\title{
FUNCTIONS STARLIKE WITH RESPECT TO OTHER POINTS
}

\author{
S. ABDUL HALIM \\ Dept. of Mathematics \\ University of Malaya \\ 59100 Kuala Lumpur \\ Malaysia
}

(Received September 26, 1990 and in revised form March 5, 1991)

ABSTRACT. In [7], Sakaguchi introduce the class of functions starlike with respect to symmetric points. We extend this class. For $0 \leqslant \beta<1$, let $S_{S}^{*}(\beta)$ be the class of normalised analytic functions $f$ defined in the open unit disc $D$ such that $\operatorname{Re} \mathrm{zf}^{\prime}(\mathrm{z}) /\left(\mathrm{f}(\mathrm{z})-\mathrm{f}^{\prime}(-\mathrm{z})\right)>\beta$, for some $\mathrm{z} \in \mathrm{D}$. In this paper, we introduce 2 other similar classes $S_{C}^{*}(\beta), S_{S C}^{*}(\beta)$ as well as give sharp results for the real part of some function for $f \in S_{S}^{*}(\beta), S_{C}^{*}(\beta)$ and $S_{S C}^{*}(\beta)$. The behaviour of certain integral operators are also considered.

KEY WORDS AND PHRASES. Starlike functions of order $B$, functions starlike with respect to symmetrical points, close-to-convex, integral operators.

AMS Subject Classification (1985 Revision): Primary 30 C 45.

1. INTRODUCTION.

Let $S$ be the class of analytic functions $f$, univalent in the unit disc $D=\{z:|z|<1\}$, with

$$
f(z)=z+\sum_{n=2}^{\infty} a_{n} z^{n} .
$$

For $0 \leqslant \beta<1$, denote by $S^{*}(\beta)$, the class of starlike functions of order $\beta$. Then $f \varepsilon S^{*}(\beta)$ if, and only if, for $z \in D$,

$$
\operatorname{Re}\left(\frac{z f^{\prime}(z)}{f(z)}\right)>B
$$

Ir [7], Sakaguchi introduced the class $S_{S}^{*}$ of analytic functions $f$, normalised by (1.1) which are starlike with respect to symmetrical points. We begin by defining the class $S_{S}^{*}$, which is contained in $K$, the class of close-to-convex functions. DEFINITION 1 .

A function $f \in S_{S}^{*}$ if, and only if, for $z \in D$,

$$
\operatorname{Re}\left(\frac{z f^{\prime}(z)}{f(z)-f(-z)}\right)>0 \text {. }
$$


DEFINITION 2.

A function $f$ with normalisations (1.l) is said to be starlike of order $B$, with respect to symmetric points if, and only if, for $z \varepsilon D$ and $0 \leqslant B<1$,

$$
\operatorname{Re}\left(\frac{2 z f^{\prime}(z)}{f(z)-f(-z)}\right)>\beta \text {. }
$$

We denote this class by $S_{S}^{*}(\beta)$ and note that $S_{S}^{*}=S_{S}^{*}(0)$.

In the same manner, we define the following new classes of close-to-convex functions, which are generalisations of the classes in El-Ashwah and Thomas [2]. DEFINITIION 3.

A function $f$ normalised by (1.1) is said to be starlike of order $B$, with respect to conjugate points if, and only if, for $z \in \cdot D$ and $0 \leqslant \beta<1$,

$$
\operatorname{Re}\left(\frac{2 z f^{\prime}(z)}{f(z) \overline{f(\bar{z})}}\right)>B \text {. }
$$

We denote this class by $S_{C}^{*}(\beta)$.

DEFINITION 4.

A function $f$ normalised by (1.1) is said to be starlike of order $B$, with respect to symmetric conjugate points if, and only if, for $z \in D$ and $0 \leqslant \beta<1$,

$$
\operatorname{Re}\left(\frac{2 z f^{\prime}(z)}{f(z) \overline{f(-\bar{z})}}\right)>\beta \text {. }
$$

We denote this class by $S_{S C}^{*}(\beta)$.

REMARK.

The class $S_{S}^{*}$ has been studied by several authors, (eg. Wu [9] and Stankiewicz [8]). For $f \in S_{S}^{*}(\beta)$, Owa et al [4] proved that for $\frac{1}{4} \leqslant \beta<\frac{1}{2}$,

$$
\operatorname{Re}\left(\frac{f(z)-f(-z)}{z}\right)>\frac{2}{3-4 B}, \quad z \in D \text {. }
$$

2. RESULTS.

THEOREM 1.

Let $f \in S_{S}^{*}(\beta)$, then for $z=r e^{i \theta} \varepsilon . D$,

$$
\operatorname{Re}\left(\frac{f(z)-f(-z)}{z}\right)^{1 /(1-\beta)} \geqslant \frac{2^{1 /(1-\beta)}}{1+r^{2}}>2^{\beta /(1-\beta)} .
$$

The result is sharp for $f_{0}$ given by

$$
f_{0}(z)-f_{0}(-z)=2 z\left(1+z^{2}\right)^{\beta-1} \text {. }
$$

To prove Theorem 1., we first require the following lemma.

LEMMA 1.

Let $g \in S^{*}(\beta)$ and be odd. Then for $z=r e^{i \theta} \varepsilon D$,

$$
\operatorname{Re}\left(\frac{g(z)}{z}\right)^{1 /(1-\beta)} \geqslant \frac{1}{1+r^{2}} \text {. }
$$


PROOF OF LEMMA.

Pinchuk [5] showed that if $F \in S^{*}(B)$, then for $z=r e^{i \theta} \varepsilon D$,

$$
\left|\left(\frac{z}{F(z)}\right)^{1 / 2(1-\beta)}-1\right| \leqslant r .
$$

Since $g$ is odd, we may write $[g(z)]^{2}=F\left(z^{2}\right)$, so that (2.1) gives

$$
\left|\left(\frac{z}{g(z)}\right)^{1 /(1-\beta)}-1\right| \leqslant r^{2} \text {, }
$$

where on squaring both sides, gives

$$
\left(\frac{r}{\lg (z))}\right)^{2 /(1-\beta)}-2 \operatorname{Re}\left[\left(\frac{z}{g(z)}\right)^{1 /(1-\beta)}\right]+1 \leq r^{4} .
$$

Thus

$$
\begin{aligned}
2 \operatorname{Re}\left[\overline{\left(\frac{g(z)}{z}\right)}^{1 /(1-\beta)}\right] & \geqslant\left(1-r^{4}\right)\left(\frac{\lg (z) \mid}{r}\right)^{2 /(1-\beta)}+1 \\
& \geqslant \frac{1-r^{4}}{\left(1+r^{2}\right)^{2}}+1
\end{aligned}
$$

where we have used the inequality [6]

$$
|g(z)| \geq \frac{r}{\left(1+r^{2}\right)^{1-\beta}}
$$

for odd starlike functions of order $B$.

The Lemma now follows at once.

PROOF OF THEOREM 1.

Since $f \in S_{S}^{*}(\beta)$, it follows that we may write

$$
g(z)=\frac{f(z)-f(-z)}{2},
$$

for $g$ an odd starlike function of order $B$. An application of Lemma 1 proves the Theorem.

Results analogous to Theorem $I$ can also be found for the classes $S_{C}^{*}(\beta)$ and $S_{S C}^{*}(\beta)$. THEOREM 2.

$$
\begin{aligned}
\text { Let } & f \in S_{c}^{*}(\beta) . \text { Then for } z=r e^{i \theta} \in D, \\
& \operatorname{Re}\left(\frac{f(z)+\overline{f(\bar{z})}}{z}\right)^{1 / 2(1-\beta)} \geqslant \frac{(\sqrt{2})^{1 /(1-\beta)}}{1+r}>2^{(2 \beta-1) / 2(1-\beta)} .
\end{aligned}
$$

The result is sharp for $f(z)+\overline{f(\bar{z})}=2 z(1+z)^{2(\beta-1)}$

PROOF

Since $f \in S_{C}^{*}(\beta)$, it is easy to see that, if

$$
F(z)=\frac{f(z)+\overline{f(\bar{z})}}{2}
$$

then $F \in S^{*}(B)$. Using the same techniques as in the proof of Lemma 1 , it follows from (2.1) that 


$$
\operatorname{Re}\left(\frac{F(z)}{z}\right)^{1 / 2(1-\beta)} \geq \frac{1}{1+r}
$$

The result now follows immediately.

Similarly, we have the following result, which we state without proof. THEOREM 3.

$$
\begin{aligned}
& \text { Let } f \quad S_{S C}^{*}(\beta) . \text { Then for } z=r e^{i \theta} \varepsilon D, \\
& \operatorname{Re}\left(\frac{f(z)-\overline{f(-\bar{z})}}{z}\right)^{1 / 2(1-\beta)} \geqslant \frac{2^{1 / 2(1-\beta)}}{1+r}>2^{(2 \beta-1) / 2(1-\beta) .}
\end{aligned}
$$

The result is sharp for $f(z)-\overline{f(-\bar{z})}=2 z(1+z)^{2(\beta-1)}$

We now consider the results of some integral operators. In [1] Das and Singh, obtained analogous results of the Libera integral operator. They proved that for $f \varepsilon S_{S}^{*}(0)$, the function $h$ given by

$$
h(z)=\frac{1}{2} \int_{0}^{z} t^{-1}[f(t)-f(-t)] d t
$$

also belongs to $\mathrm{S}_{\mathrm{S}}^{*}(0)$.

The result below generalises that of Das and Singh.

THEOREM 4.

Let $f \in S_{S}^{*}(\beta)$. Then the function $H$ defined by

$$
H(z)=\frac{a+1}{2 z^{a}} \int_{0}^{z} t^{a-1}[f(t)-f(-t)] d t,
$$

also belongs to $S_{S}^{*}(\beta)$ for $z \in D$ and $a+\beta>0$.

We first require the following Lemma due to Miller and Mocanu [5].

LEMMA 2.

Let $M$ and $N$ be analytic in $D$ with $M(0)=N(0)=0$ and let $B$ be any real number. If $\mathrm{N}(\mathrm{z})$ maps $\mathrm{D}$ onto a (possibly many sheeted) region which is starlike with respect to the origin, then for $z \in D$,

$$
\operatorname{Re} \frac{M^{\prime}(z)}{N^{\prime}(z)}>\beta \Rightarrow \operatorname{Re} \frac{M(z)}{N^{\prime}(z)}>\beta,
$$

and

$$
\operatorname{Re} \frac{M^{\prime}(z)}{N^{\prime}(z)}<\beta \Rightarrow \operatorname{Re} \frac{M(z)}{N(z)}<\beta .
$$

PROOF OF THEOREM 4.

$$
\begin{aligned}
& \text { (2.2) gives, } \begin{aligned}
\frac{2 z H^{\prime}(z)}{H(z)-H(-z)} & =\frac{z^{a}[f(z)-f(-z)]-a \int_{0}^{z} t^{a-1}[f(t)-f(-t)] d t}{\int_{0}^{z} t^{a-1}[f(t)-f(-t) d t} \\
& =\frac{M(z)}{N(z)} \text {, say. } \\
\text { Note that } M(0) & =N(0)=0 \text { and for } f \varepsilon S_{S}^{*}(B),
\end{aligned}
\end{aligned}
$$




$$
\begin{aligned}
\operatorname{Re}\left(1+\frac{z N^{\prime \prime}(z)}{N^{\prime}(z)}\right) & =a+\operatorname{Re}\left(\frac{z\left[f^{\prime}(z)+f^{\prime}(-z)\right]}{f(z)-f(-z)}\right) \\
& >a+B
\end{aligned}
$$

Thus $N(z)$ is starlike if, and only if $a>-\beta$.

Furthermore, since

$$
\operatorname{Re} \frac{M^{\prime}(z)}{N^{\prime}(z)}=\operatorname{Re}\left(\frac{z\left[f^{\prime}(z)+f^{\prime}(-z)\right]}{f(z)-f^{\prime}(-z)}\right)>B
$$

Lemma 2 shows that $H \in S_{S}^{*}(B)$.

Finally, we give the following analogous results for the classes $S_{C}^{*}(B)$ and $S_{S C}^{*}(B)$. THEOREM 5.

Let $f \in S_{c}^{*}(\beta)$. Then $H$ defined by

$$
H(z)=\frac{a+1}{2 z^{a}} \int_{0}^{z} t^{a-1}[f(t)+\overline{f(\bar{t})}] d t,
$$

also belongs to $S_{c}^{*}(\beta)$ for $z \in D$ and $a+\beta>0$.

PROOF.

Since $f \in S_{C}^{*}(B),(2.3)$ gives

$$
\begin{aligned}
\overline{\int_{0}^{\bar{z}} t^{a-1}[f(t)+\overline{f(\bar{t})}] d t} & =2 \int_{0}^{\bar{z}} t^{a-1}\left(t+\sum_{n=2}^{\infty} \operatorname{Re} a_{n} t^{n}\right) d t \\
& =2 \int_{0}^{z} t^{a-1}\left(t+\sum_{n=2}^{\infty} \operatorname{Re} a_{n} t^{n}\right) d t \\
& =\int_{0}^{z} t^{a-1}[f(t)+\overline{f(\bar{t})] d t .}
\end{aligned}
$$

Thus

$$
\begin{aligned}
\frac{2 z H^{\prime}(z)}{H(z)+\overline{H(\bar{z})}} & =\frac{z^{a}[f(z)+f(\bar{z})]-a \int_{0}^{z} t^{a-1}[f(t)+\overline{f(\bar{t})}] d t}{\int_{0}^{z} t^{a-1}[f(t)+\overline{f(\bar{t})] d t}} \\
& =\frac{M(z)}{N(z)},
\end{aligned}
$$

where $M(0)=N(0)=0$ and $N \in S^{*}$ for $a+B>0$.

On using Lemma 2 it follows that $H \in S_{C}^{*}(\beta)$.

THEOREM 6.

Let $f \in S_{S C}^{*}(\beta)$. Then $H$ defined by

$$
H(z)=\frac{a+1}{2 z^{a}} \int_{0}^{z} t^{a-1}[f(t)-\overline{f(-\bar{t})]} d t,
$$

also belongs to $S_{S c}^{*}(\beta)$ for $z \in D$ and $a+\beta>0$. 
PRDOF.

$$
\begin{aligned}
& \text { For } f \in S_{S C}^{*}(\beta),(2.4) \text { gives } \\
& \overline{H(-\bar{z})}=\frac{a+1}{(-z)^{a}} \overline{\int_{0}^{-\bar{z}} t^{a-1}[f(t)-\overline{f(-\bar{t})}] d t} \\
& =\frac{a+1}{(-z)^{a}}\left\{\frac{2(-z)^{a+1}}{a+1}+\sum_{n=2}^{\infty} \frac{(-z)^{n+a}}{n+a}\left(\overline{a_{n}}+(-1)^{n+1} a_{n}\right)\right\} \\
& =\frac{-(a+1)}{z^{a}} \int_{0}^{z} t^{a-1}[f(t)-\overline{f(-\bar{t})}] d t .
\end{aligned}
$$

As before, writing

$$
\frac{2 z H^{\prime}(z)}{H(z)-\overline{H(-\bar{z})}}=\frac{M(z)}{N(z)},
$$

one can show that $N \in S^{*}$ and hence using Lemma 2 the result follows.

\section{REFERENCES}

1. R.N.Das and P.Singh, On subclasses of schlicht mapping, Indian J. Pure Appl. Math., 8, (1977), 864-872.

2. R.M.EI-Ashwah and D.K.Thomas, Some subclasses of close-to-convex functions, J. Ramanujan Math. Soc., 2(1), (1987), 85-100.

3. S.S.Miller and P.T.Mocanu, Second order differential inequalities in the complex plane, J. Math. Ana. Appl., 65, (1978), 289-304.

4. S.Owa, Z.Wu and F.Ren, A note on certain subclass of Sakaguchi functions, Bull. de la Royale de Liege, 57( 3), (1988), 143-150.

5. B.Pinchuk, On starlike and convex functions of order B, Duke Math. Journal, 35, (1968), 721-734.

6. M.S.Robertson, On the theory of univalent functions, Ann. Math., 37, (1936), $374-408$.

7. K.Sakaguchi, On a certain univalent mapping, J. Math. Soc. Japan, 11, (1959), $72-75$.

8. J.Stankiewicz, Some remarks on functions starlike w.r.t. symmetric points, Ann. Univ. Marie Curie Sklodowska, 19(7), (1965), 53-59.

9. Z.Wu, On classes of Sakaguchi functions and Hadamard products, Sci. Sinica Ser. A, 30, (1987), 128-135. 


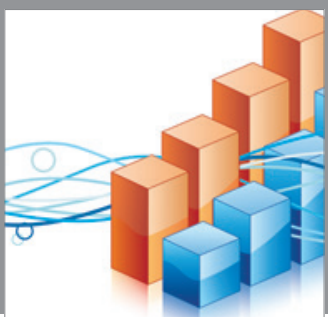

Advances in

Operations Research

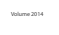

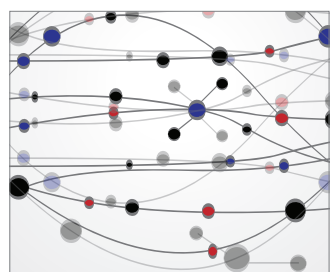

\section{The Scientific} World Journal
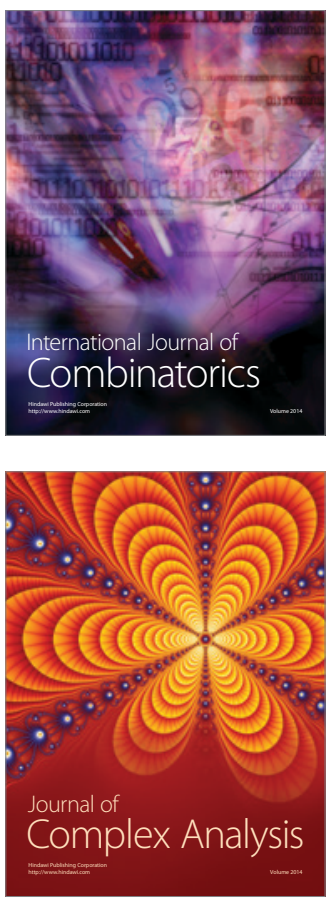

International Journal of

Mathematics and

Mathematical

Sciences
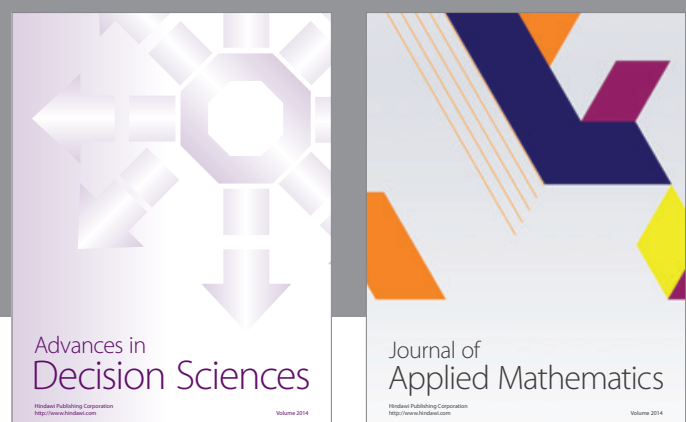

Journal of

Applied Mathematics
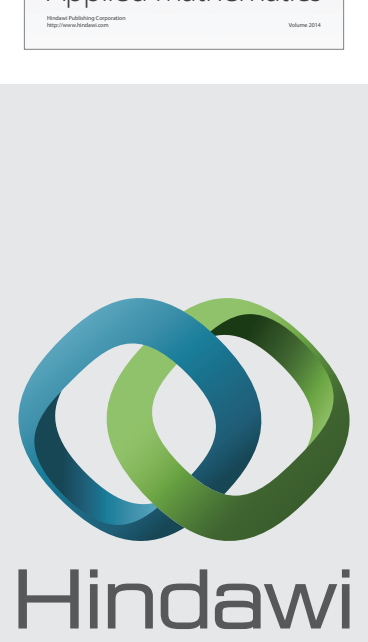

Submit your manuscripts at http://www.hindawi.com
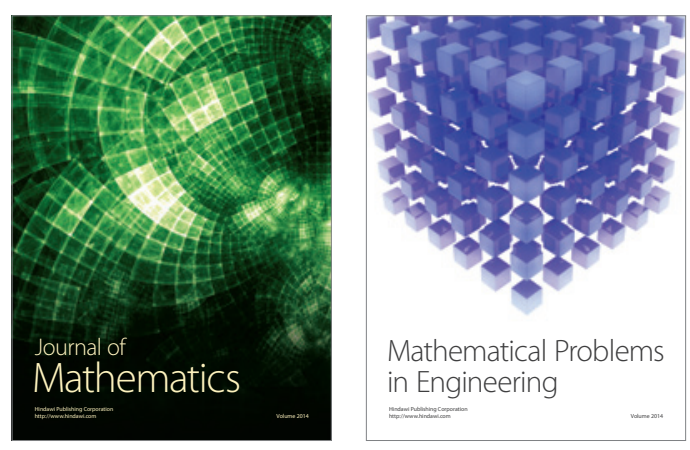

Mathematical Problems in Engineering
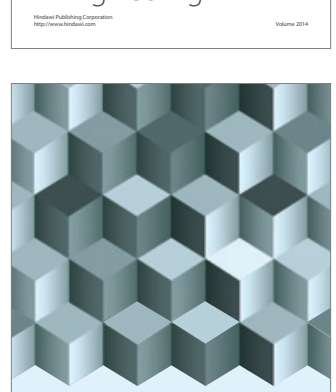

Journal of

Function Spaces
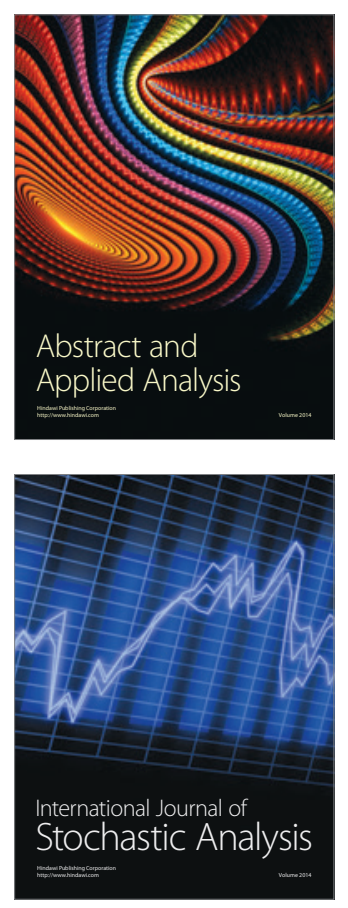

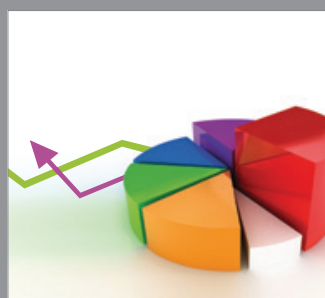

ournal of

Probability and Statistics

Promensencen
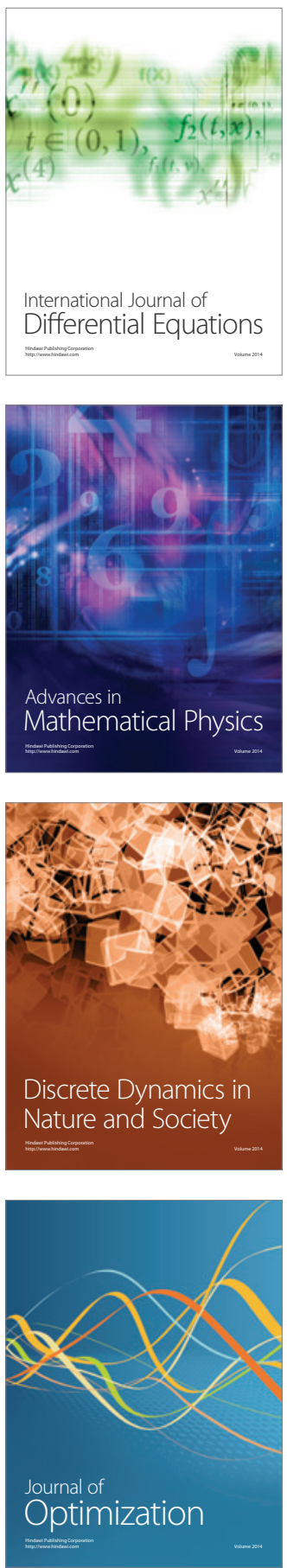